\title{
Frequency Response Optimization of Saw Filter using Canonical Genetic Algorithm
}

\author{
Prachi Chaudhary \\ ECE Dept., Deenbandhu \\ Chhotu Ram University of Sci. \\ \& Tech., Murthal, Haryana \\ (INDIA).
}

\author{
Priyanka \\ ECE Dept., Deenbandhu \\ Chhotu Ram University of Sci. \\ \& Tech., Murthal, Haryana \\ (INDIA).
}

\author{
Manoj Duhan \\ ECE Dept., Deenbandhu \\ Chhotu Ram University of Sci. \\ \& Tech., Murthal, Haryana, \\ (INDIA).
}

\begin{abstract}
An optimization program is employed to design the inter-digital transducer (IDT) structure of the surface acoustic wave (SAW) transducer to aim at improvement in the transducer characteristics and to improve the frequency response of SAW filter. In this paper, Genetic algorithm is used to optimize the response of a SAW filter. Then, the comparison of the response of SAW filter is done by using GA and without GA. The goal of optimization is to find best value for each variable in order to achieve minima or maxima of an objective function within the given constraints. In present study, three design variables of SAW filter i.e. number of finger pairs in input IDT $\left(\mathrm{Np}_{1}\right)$, number of finger pairs in `output IDT $\left(\mathrm{Np}_{2}\right)$, center to center distance between two electrodes in a finger pair (d), are optimized. After getting the optimized parameters of SAW filter, the frequency response of bandpass SAW filter is obtained, which is better as compared to the frequency response of SAW filter getting from other methods in terms of bandwidth and ripples amplitude.
\end{abstract}

Keywords: surface acoustic wave (SAW) Filter, Optimization, Genetic Algorithm (GA), Inter-digital Transducer (IDT).

\section{INTRODUCTION}

Surface Acoustic Wave (SAW) devices have been used since the early 1990s as critical components in many radio frequency (RF) transmitters and receivers due to their small size, efficient operation, and excellent performance characteristics [13]. Inter-digital Transducer (IDT) is a comb-shaped structure fabricated over the piezoelectric substrate. When the sinusoidal voltage is applied to the input IDT then comb shape of electrode in IDT sets up electric field along the surface of the crystal. Change in electric field cause the crystal to expand and contract [16]. A voltage applied to the IDT produces dynamic strains in the substrate and initiates elastic waves that travel along the surface. Only the frequencies with a whole no. of wavelength between the teeth of each comb will propagate across the comb fingers. Other frequencies will be diminished because of destructive interference, which results in filter [12]. The frequency response characteristics of SAW filters are governed primarily by their geometrical structures, namely, the configurations of InterDigital Transducers (IDTs) and Shorted Metal Strip Array (SMSA) reflectors fabricated on piezoelectric substrates. Therefore, in order to obtain desirable frequency response characteristics with SAW filters, we have to decide their suitable structure. For realizing a desirable bandpass filter, several computer-aided design approaches have been reported in the literature [12]. However, even if the structural design of SAW filters is formulated as an optimization problem [14, 15] most design techniques have relied on local optimization methods. For example, a common design technique based on the delta-function model of IDT employed an iterative algorithm to yield an optimum SAW filter. We also applied a local search to the optimum design problem of a resonator type SAW filter [10], where we used the least equivalent circuit model of IDT. Unfortunately, we could rarely obtain a globally optimal or near-optimal structure of the SAW filter only by the local search, because the local optimization method doesn't have the ability to survey a vast region of the search space $[8,9,11]$. In gradient based methods typically used in optimization, the final result can depend strongly on the initial guess of the parameters. Furthermore, the gradient method can converge to a local minimum, and often there is no way of determining whether the solution is the global minimum or not, or how good the quality of the final solution is compared to the global minimum. Performing several optimization runs with different points can help in finding a better solution, but this kind of manual iteration is often time-consuming. Stochastic optimization methods, such as Evolutionary Algorithms (EAs) [1], Genetic Algorithms (GAs) [5] depend on randomness in the search process. The algorithms aim at searching over the whole population space, starting from a random situation and converging towards the global minimum. Each iteration step is based on the solutions created in the previous step, but the method of proceeding from one step to the next is in some way arbitrary. The random element increases the probability of finding the global minimum. Even though there is no guarantee of converging to the global minimum, the final solution is not dependent on the initial guess of parameters. Therefore, the problem of converging to a low-quality local optimum near the starting point of the optimization process, often associated with gradient optimization methods, can be overcome [1]. Genetic optimization has been previously studied in the context of transducer optimization [5]. In this paper, the Genetic algorithm is applied to optimize the response of a SAW filter. In this paper, a comparison between the response of SAW filter without GA and the response using GA is given. 


\section{GENETIC ALGORITHM}

2.1. Overview: We can use the concept of natural evolution to solve problems in the computer. Possible solutions make up the population and better solutions, equivalent to fitter organisms in nature, are more likely to reproduce and pass on their genetic information to the next generation. Over time we could expect that good solutions are evolved just as organisms have evolved in nature [3]. To solve a problem using genetic algorithms, otherwise known as evolutionary computing, we need the following:

1. A system of encoding the possible solutions or chromosome structure.

2. An initial population of solutions.

3. A function to evaluate the solution fitness.

4. A method of selecting solutions to be used to produce new solutions.

5. Recombination and Mutation operators to create new solutions from those existing.

In order to find desirable SAW filters' structures, the design of them is formulated as an optimization Problem.

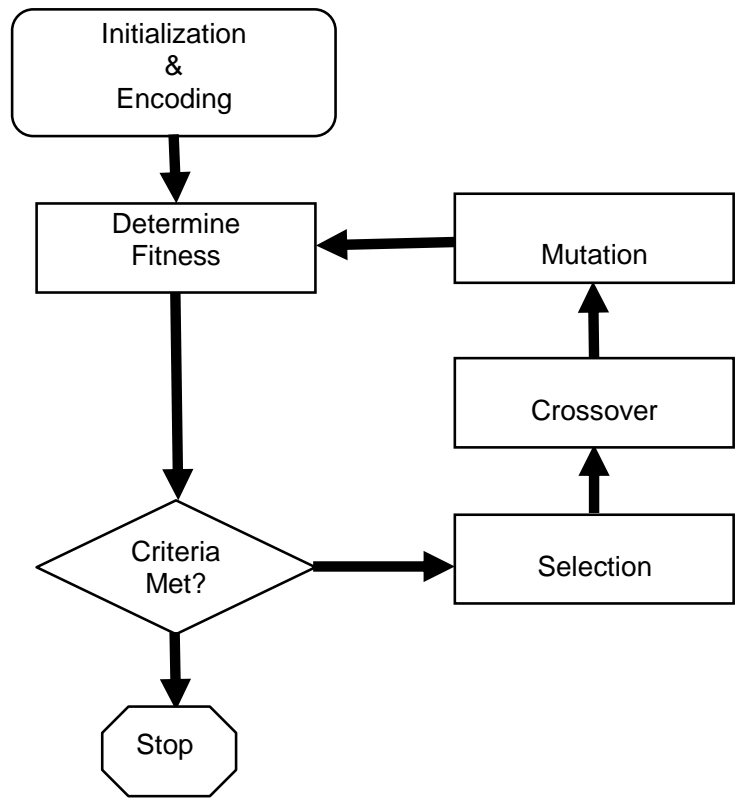

Fig.1: Flowchart of Canonical Genetic Algorithm.

The GA algorithm process is discussed through the GA flow chart in Fig. 1. Through the evaluation of objective function, individual quality is assessed. Mutation is performed to one individual to produce a new version of it where some of original genetic material has been randomly changed. Selection process help to decide which individuals are to be used for reproduction and mutation in order to produce new search points.

2.2 GA used in optimization: In this paper, we think about an optimal configuration of a three-IDT type SAW filter in Fig. 2. The SAW filter consists of five components: one receiver IDT (IDT-I), two transmitter IDTs (IDT-2) and two reflectors realized by shorted metal strip arrays (SMSA). Each IDT comprises some pairs of electrodes which are sometimes called fingers, while SMSA has numerous metal strips connected electrically in parallel. These components of the SAW filter are arranged bilateral symmetry on a piezoelectric substrate [8].

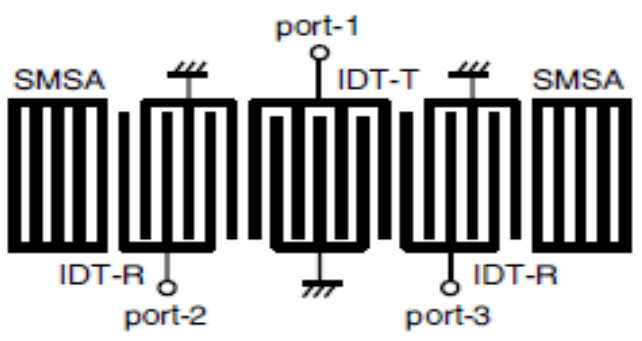

Fig. 2: Three IDT type SAW filter [6, 8]

Problem space [8]:

$$
\begin{aligned}
& X i[\underline{X i}, \overline{X i}] \text { design parameter } \\
& X_{0}[200,400] \text { overlap of electrodes } \\
& X_{1}[10.0,20.0] \text { fingers' number of IDT-R } \\
& X_{2}[15.5,25.5] \text { ditto of IDT-T } \\
& X_{3}[1.0,3.0] \text { ditto of modulated IDT } \\
& X_{4}[50,150] \text { strips' number of SMSA } \\
& X_{5}[0.4,0.6] \text { metallization ratio of IDT } \\
& X_{6}[0.4,0.6] \text { ditto of SMSA } \\
& X_{7}[0.91,0.92] \text { pitch ratio of IDT } \\
& X_{8}[1.00,1.05] \text { ditto of SMSA } \\
& X_{9}[1.95,2.05] \text { finger pitch of IDT } \\
& X_{10}[3900,4000] \text { thickness of electrode }
\end{aligned}
$$

Therefore, as well as conventional optimum design techniques for SAW filters, design parameters describing the structure of a SAW. For example, the numbers of IDTs' fingers, the number of SMSAs' strips, and the overlap between electrodes are included in decision variables. Also, the values of respective decision variables $X i \varepsilon X$ are bounded by their parametric limitations $[6,7,8]$. They are selected as decision variables: $X=\left(X_{1}, \cdots, X_{D}\right)$, where, $X$ denotes a set of feasible solutions, or the problem space, satisfying the conditions .

$$
\underline{X i} \leq X i \leq \overline{X i}, i=0,1 \cdots \mathrm{D}-1 .
$$

After this, we define the objective function of the optimization problem.

\section{PROBLEM FORMULATION}

We formulate the optimum design of the SAW filter mathematically. Let $F$ be the feasible region of solutions, namely a whole set of possible design parameters constrained by lower bound and upper bound. For minimizing the objective function ' $y_{a b}$ ', the structural design of the SAW filter is expressed as an optimization problem as follows.

$$
\begin{aligned}
& \text { Minimize } \mathrm{y}_{\mathrm{ab}} \\
& \text { subject to }: \mathrm{y}_{\mathrm{ab}} \& F
\end{aligned}
$$


$F$ is a set of three design variables $(\mathrm{Np} 1, \mathrm{~Np} 2, \mathrm{~d})$ which are taken to optimize the response of SAW filter.

The desirable filter characteristics of the SAW filter is specified by the transfer admittance, $y_{a b}$, where the values of ' $y_{a b}$ ' depend on three design variables viz $\mathrm{Np} 1, \mathrm{~Np} 2$ and d. Therefore, considering all criteria related to transfer admittance for the SAW filter, the objective function yab is defined as follows:

Transfer Admittance:

Magnitude response: $\mathrm{y}_{\mathrm{ab}}=\mathrm{C} *\left(\operatorname{sinc}(\mathrm{x}) *{ }^{*} \operatorname{sinc}(\mathrm{x})\right.$

$\mathrm{x}=\mathrm{f}-\mathrm{fo} ; \mathrm{x}=\mathrm{x}$. /fo; $\mathrm{x}=\mathrm{Np} 1 * \mathrm{x}$

$\mathrm{C}=8 * \mathrm{~Np} 1 * \mathrm{~Np} 2 * \mathrm{Go} 1$

Go $1=\mathrm{K} 2 *$ Cs $*$ fo

$\mathrm{Np} 1=\operatorname{randint}(1,1, \mathrm{FPIR})$

$\mathrm{Np} 2=\operatorname{randint}(1,1, \mathrm{FPOR})$

$\mathrm{d}=\operatorname{randint}(1,1, \mathrm{FOR})$

Phase response: $\mathrm{y}_{\mathrm{ab}}=\mathrm{y}_{\mathrm{ab}} *$ phase

phase $=\exp ($ thita $) ;$ thita $=$ complex $($ zerovector , delf $)$;

zerovector $=$ zeros $(1$, length $(f))$; delf $=$ onevector - delf; delf $=$ pi $*$ delf; delf = delf - phi;

onevector $=\operatorname{ones}(1$, length $(\mathrm{f})) ; \mathrm{phi}=($ separation $* \mathrm{~W} 1) . / \mathrm{Vs}$;

delf $=\mathrm{f}-$ fo $;$ delf $=$ delf.$/$ fo; delf $=(\mathrm{Np} 1+\mathrm{Np} 2) *$ delf;

separation $=\mathrm{d}-\mathrm{d} 1-\mathrm{d} 2$;

$\mathrm{d} 1=2 * \mathrm{~Np} 1 *$ lambda - lambda; $\mathrm{d} 1=\mathrm{d} 1 / 2$;

$\mathrm{d} 2=2 * \mathrm{~Np} 2 *$ lambda - lambda; $\mathrm{d} 2=\mathrm{d} 2 / 2$;

lambda $=\mathrm{Vs} /$ fo; lambda $=$ lambda/2;

\section{SIMULATION RESULTS \& COMPARISON}

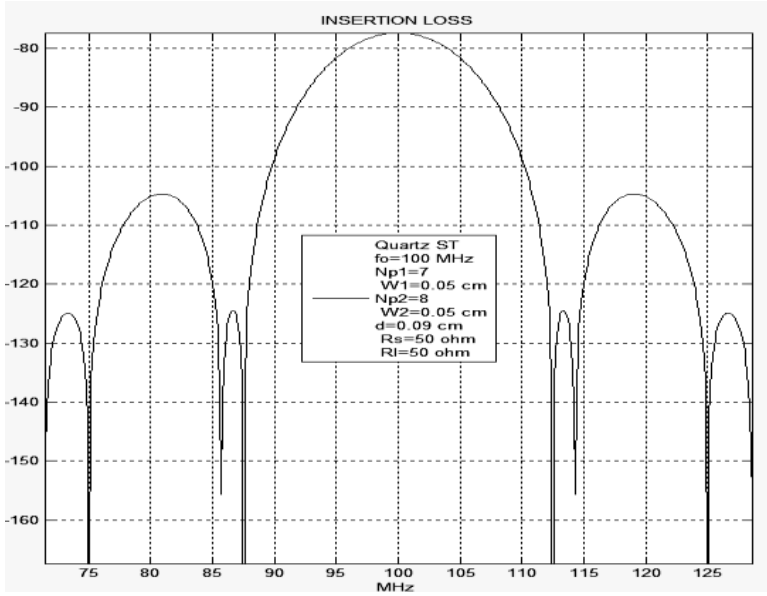

Fig. 3: SAW filter response without using GA.

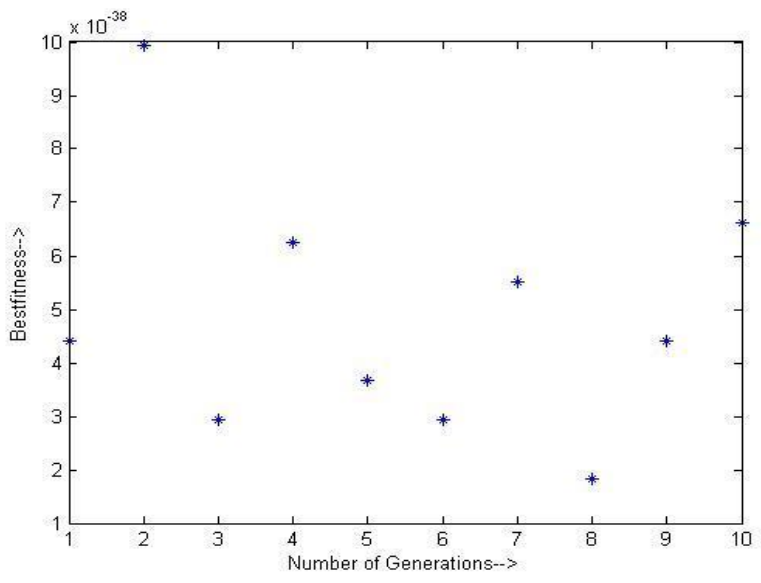

Fig. 4: Fittest solution $(\mathrm{g}=10)$.

Here the response of SAW filter is compared by using GA and without GA. Also the response of SAW filter for different generations using GA is shown.

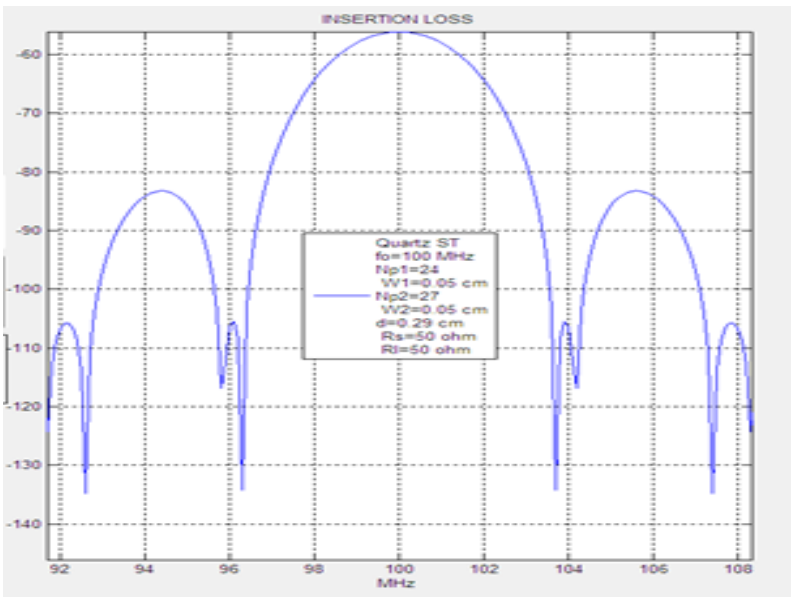

Fig. 5: Response of SAW filter with GA $(g=10)$.

In first experiment, the number of generation are 10 , in second experiment the no. of generation are 20, in third experiment no. of generation are 50 and in fourth experiment no. of generation are 100. The values of other parameters are kept fixed. In first experiment, the values are NP1 $=7, \mathrm{NP} 2=8, \mathrm{D}=9$, fig. 3, shows SAW filter response without using GA. The values of other parameters are kept fixed, In first experiment, the values are $\mathrm{NP} 1=7, \mathrm{NP} 2=8, \mathrm{D}=9$, fig. 3, shows SAW filter response without using GA. The values of other parameters are kept fixed, which are $\mathrm{F}_{\mathrm{o}}=100 \mathrm{Mhz}, \mathrm{W}_{1}=$ $0.05 \mathrm{~cm}, \mathrm{~W}_{2}=0.05 \mathrm{~cm}, \mathrm{R}_{\mathrm{s}}=50 \mathrm{ohm}, \mathrm{R}_{\mathrm{L}}=50 \mathrm{ohm}$.

Now we are going to obtain response of saw filter using Genetic Algorithm. When we give NP $1=$ [5 15], NP2 = [ 5 15], D = [2 10], and no. of generation $=10$, the optimized values areNP1 $=13, \mathrm{NP} 2=$ $15, \mathrm{~d}=0.1$. The best fittest solution is obtained and is shown in fig. 4 . 
Then, we took No. of generation $=20$

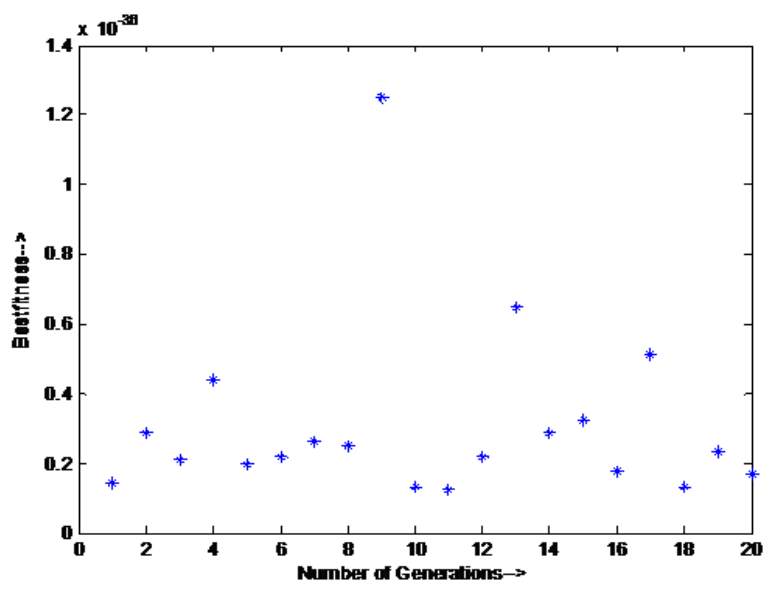

Fig. 6: Fittest solution $(\mathrm{g}=20)$.

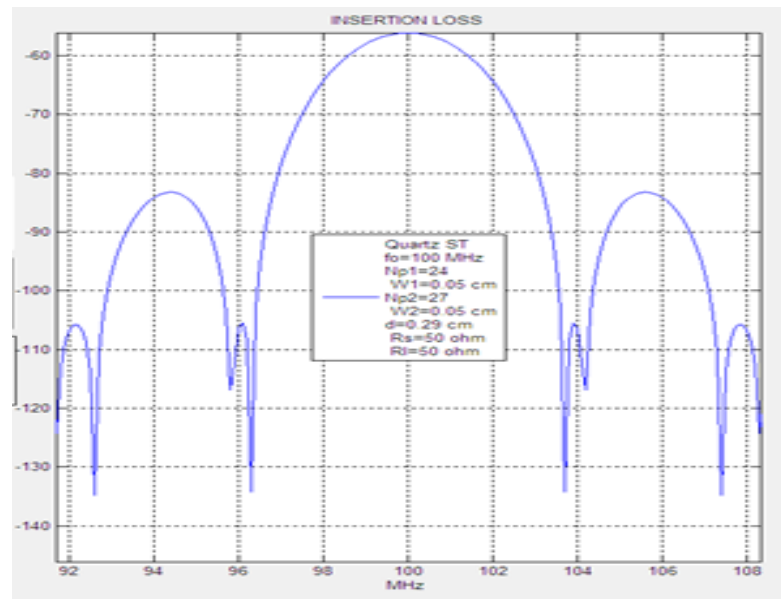

Fig. 7: Response of SAW filter with GA ( $g=20)$.

Then, we took no. of generation $=50$

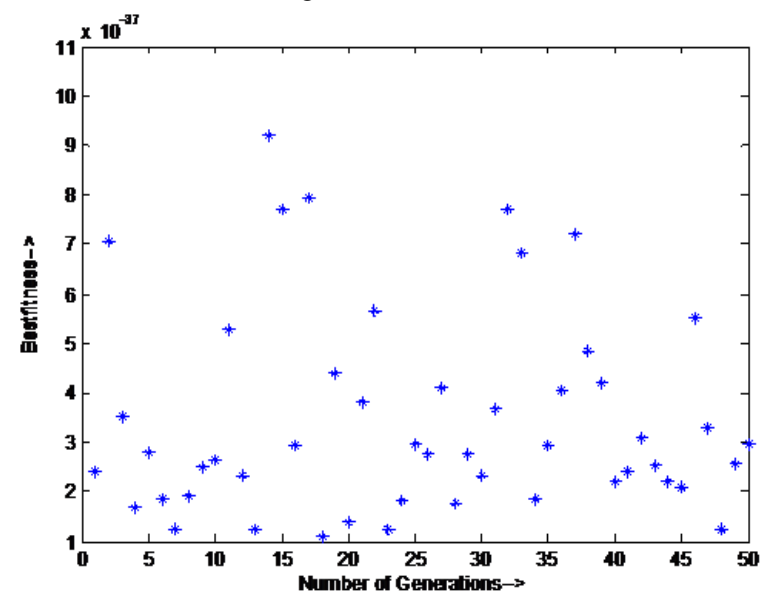

Fig. 8: Fittest solution $(\mathrm{g}=50)$.

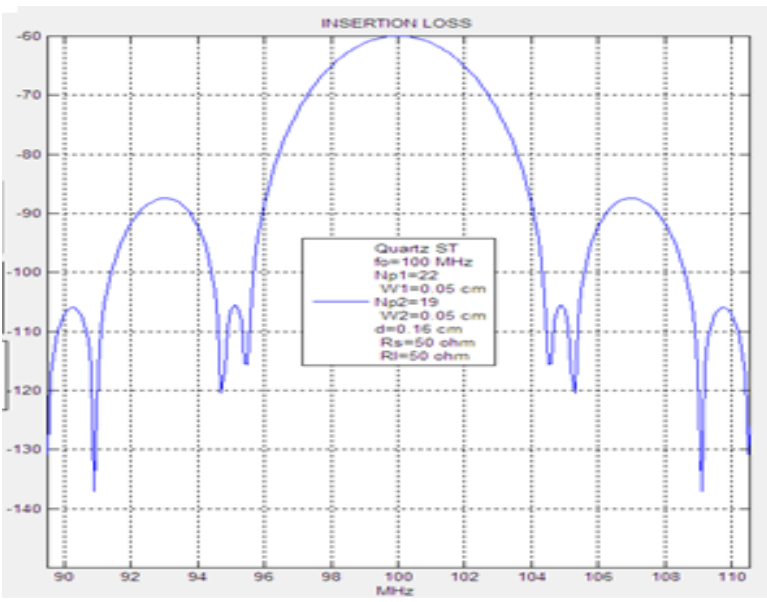

Fig. 9: Response of SAW filter with GA $(g=50)$.

Then, we took no. of generation $=100$

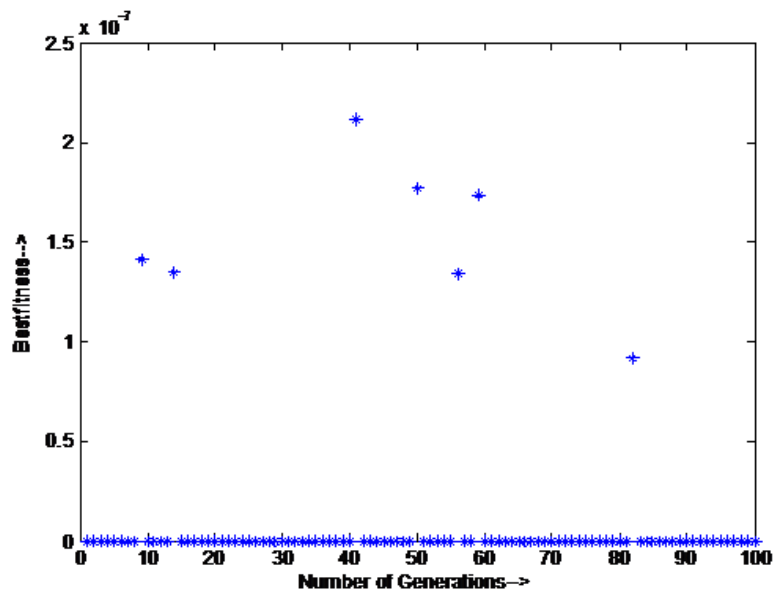

Fig.10: Fittest solution $(\mathrm{g}=100)$.

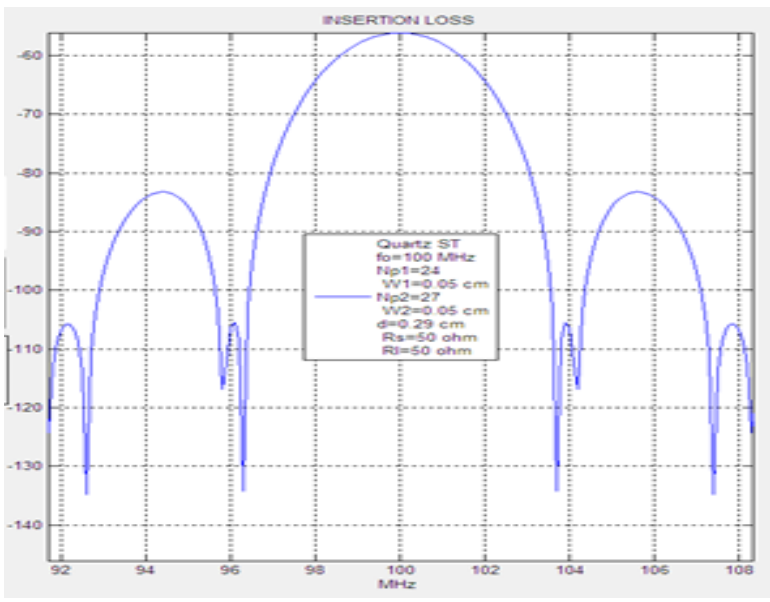

Fig.11: Response of SAW filter with GA ( $g=100)$. 
It is clear from the above comparison that response of SAW filter using Genetic Algorithm is better than the response obtained without using GA .In first experiment we take no. of generation $=10$, Then we have increased the no. of generations and compare the results of each generation. In each generation, we randomly generate a range of value of each parameter viz. NP1, NP2 and d. After analysis, we found that frequency responses of SAW filter using GA are better as compared to response without GA. With the increase in no. of generation in GA, the response is improving but computation time increase with increase in generation, so we should put an eye on number of generations also. After analysis, we found that frequency responses of SAW filter using GA are better as compared to response without GA.

\section{CONCLUSION \& FUTURE DIRECTIONS}

In the presented method of optimization of frequency response of SAW filter using genetic algorithm, the bandwidth is improved, the number and amplitude of ripples is reduced as compared to other method of optimization. The experimental results clearly indicate that GA gives better results as compared to results obtained without GA. It is effective to use the optimization program to design the IDT structure of the SAW transducer to aim at improvement in the transducer characteristics and to improve the frequency response of SAW filter.

Future work on the optimization of frequency response of SAW filter could be carried out by developing another objective function. Particle swarm optimization (PSO) algorithm can be used for optimization of frequency response of SAW filter. Another possibility for future work is to develop a GA based GUI for designing and optimizing SAW filter.

\section{REFERENCES}

[1] Kiyoharu Tagawa, "Evolutionary Computation Techniques for the Optimum Design of Balanced Surface Acoustic Wave Filters" proc. in", in Proc. of the 2008 IEEE International Ultrasonics, Ferroelectrics

[2] Guilling Huang, Qida Zhao, Luming Zhao" optimization of SAW transducer design by probabilistic global search lausanne",Proc. in electromagnetic research symposium Hangzhou China, March 2008.

[3] S.N.Sivanandam, S.N.Deepa, "Introduction to Genetic Algorithms”, Springer-Verlag, Berlin, 2008.

[4] K. Tagawa, M. Masuoka, and M. Tsukamoto, "Robust optimum design of SAW filters with the Taguchi method and a memetic algorithm", in Proc. of the 2005
IEEE Congress on Evolutionary Computation, pp.21462153.

[5] J. Meltaus, P. Hämäläinen, V. P. Plessky, and M. M. Salomaa, "Genetic optimization algorithms in the design of coupled SAW filters", in Proc. of the 2004 IEEE Ultrasonics Symposium, pp. 1901-1904.

[6] K. Tagawa, T. Ohtani, T. Igaki, and S. Seki, "Robust optimization design of SAW filters by using penalty function method", in Proc. of the 2004 IEEE International Conference on Industrial Technology, pp. 751-756.

[7] S. Goto and T. Kawakatsu, "Optimization of the SAW filter design by immune algorithm", in Proc. of the 2004 IEEE International Ultrasonics, Ferroelectrics and Frequency Control Joint $50^{\text {th }}$ Anniversary Conference, pp. 600-603.

[8] K. Tagawa, T. Yamamoto, T. Igaki, and S. Seki, "An Imanishian Genetic Algorithm for the Optimum Design of Surface Acoustic Wave Filter", in Proc. of the 2003 Congress on Evolutionary Computation, v.4, pp. 27482755 .

[9] Lee, Y., S. Lee, and Y. Roh, "Design of withdrawalweighted SAW filters", IEEE Transactions on Ultrasonics, Ferroelectrics and Frequency Control, Vol. 49, No. 3, 337-344, March 2002.

[10] K. Tagawa, K. Togunaka, H. Haneda, T. Igaki, and S. Seki, "Optimal design of three-IDT type SAW filter using local search", in Proc. of the 2002 IEEE 28th Annual Conference of the Industrial Electronics Society, pp. 2572-2577.

[11] V. Prabhu, B. S. Panwar and Priyanka, "Linkage learning genetic algorithm for the design of withdrawal weighted SAW filters", Proc. of IEEE Ultrasonics Symposium, pp. 357-360, 2002.

[12] K.-Y. Hashimoto, Surface Acoustic Wave Devices in Telecommunications: Modelling and Simulation, Springer-Verlag, Berlin, 2000.

[13] C. K. Campbell, Surface Acoustic Wave Devices for Mobile and Wireless Communications, Academic Press, 1998.

[14] J. Franz, C. C. W. Ruppel, F. Seifert and R. Weigel, "Hybrid optimization techniques for the design of SAW filters", Proc. of IEEE Ultrasonics Symposium, 1997, pp. 33-36.

[15] C. C. W. Ruppel, A. A. Sachs, and F. J. Seifert, “A review of optimization algorithms for the design of SAW transducers", in Proc. Of 1991 IEEE Ultrasonics Symposium, pp. 73-83.

[16] Rayleigh, L., On waves propagated along the plane surface of elastic solid," Proc. London Math. Society, Vol. 17, 4\{11, 1885\}. 